\title{
Role of Neuroimaging in Guiding Treatment Decisions on Endovascular Thrombectomy
}

\author{
(ㅇ)(ㅇ)(요 \\ Authors \\ Daniel Kaiser ${ }^{1}$, Johannes C. Gerber ${ }^{1}$, Volker Puetz ${ }^{2}$ \\ Affiliations \\ 1 Institute for Diagnostic and Interventional Neuroradiology, \\ University Hospital Carl Gustav Carus Dresden \\ 2 Clinic and Polyclinic for Neurology, University Hospital Carl \\ Gustav Carus Dresden \\ Key words \\ stroke, neuroimaging, endovascular thrombectomy \\ Bibliography \\ DOI http://dx.doi.org/10.1055/s-0043-102381 \\ Published online: 2017 | Neurology International Open 2017; 1: \\ E18-E27 \\ (c) Georg Thieme Verlag KG Stuttgart · New York \\ ISSN 2511-1795 \\ Correspondence \\ Daniel Kaiser \\ Institute for Diagnostic and Interventional Neuroradiology
}

\author{
University Hospital Carl Gustav Carus Dresden \\ Fetscherstr. 74 \\ 01307 Dresden \\ daniel.kaiser@uniklinikum-dresden.de
}

\begin{abstract}
Six recent randomized controlled trials showed a significant benefit of endovascular therapy on patient outcome in acute ischemic stroke due to anterior cerebral artery occlusion. The positive results of these trials need to be implemented in clinical routine. Suitable patients should be evaluated for thrombectomy reliably and fast. All trials confirmed the role of pretherapeutic neuroimaging as crucial in selecting patients who can benefit from endovascular therapy. However, different approaches have been used, including imaging of the target vascular occlusion, infarct core, arterial collateral supply or the penumbra. In this review we discuss, in the context of the recent trials, the different methods of non-invasive neuroimaging and their role in decision-making for thrombectomy in acute ischemic stroke.
\end{abstract}

\section{Introduction}

An ischemic stroke, caused by vascular occlusion resulting in reduced perfusion, is a potentially reversible disease that requires to be rapidly diagnosed and treated. Untreated, it can lead to persistent disability or death within a very short time. Over the last 2 decades, the treatment of acute arterial occlusion in stroke patients has developed into a safe and highly effective causal therapy, in parallel with progress in stroke imaging.

\section{Positive Studies on Endovascular Therapy}

In 2015, the randomized controlled multicentre trials MRCLEAN [1], ESCAPE [2], EXTEND-IA [3], SWIFT PRIME [4] and REVASCAT [5] achieved high recanalization rates in patients with acute proximal anterior circulation artery occlusion using modern thrombectomy devices. Additionally, these trials demonstrated a significant benefit of endovascular thrombectomy on patient outcome compared to evidence based standard therapy used so far, including intravenous thrombolysis alone. The randomized controlled trial THRACE recently confirmed these results [6]. With the exception of REVASCAT and ESCAPE (extended time window up to $8 \mathrm{~h}$ and $12 \mathrm{~h}$, respectively), the studies aimed at treating suitable patients within a maximum of $6 \mathrm{~h}$ at a center with experience in endovascular therapy [7]. An imaging-based confirmation of an ischemic stroke with detection of a proximal artery occlusion in the anterior circulation was the crucial requirement to select patients for end- ovascular thrombectomy in all trials. In detail, different neuroimaging approaches, including imaging of the vascular system, infarct core and penumbra (EXTEND-IA and in part SWIFT PRIME) or the arterial collateral status (ESCAPE) have been used for pretreatment patient evaluation ( $\triangleright$ Table 1 ). This seems to be partly reflected in the variance of the treatment effect compared to the control groups (absolute risk reduction: $11-31 \%$, > Table 1) [8]. The specific imaging-based inclusion criteria were determined by the results of previous randomized controlled trials of endovascular stroke therapy, such as IMS III [9], MR RESCUE [10], and SYNTHESIS EXP [11]. Although the latter did not show a benefit of endovascular thrombectomy over standard therapy, they have highlighted the important role of primary multimodal neuroimaging in predincting therapeutic effect and patient outcome [12].

The above-mentioned findings have been taken into consideration in the recommendations of recently published guidelines. The positive results of the current studies now have to be transferred to the clinical routine $[13,14]$. This requires algorithms that enable stroke patients to be selected quickly and reliably for effective and beneficial therapy.

In this review, different techniques of non-invasive neuroimaging and evaluation are explained and their implication in diagnosis of acute stroke is demonstrated. The focus is on their role in guiding treatment decision on endovascular thrombectomy. This is discussed on the basis of the current study situation. 
- Table 1 Imaging-based inclusion criteria and treatment effect of positive endovascular thrombectomy trials.

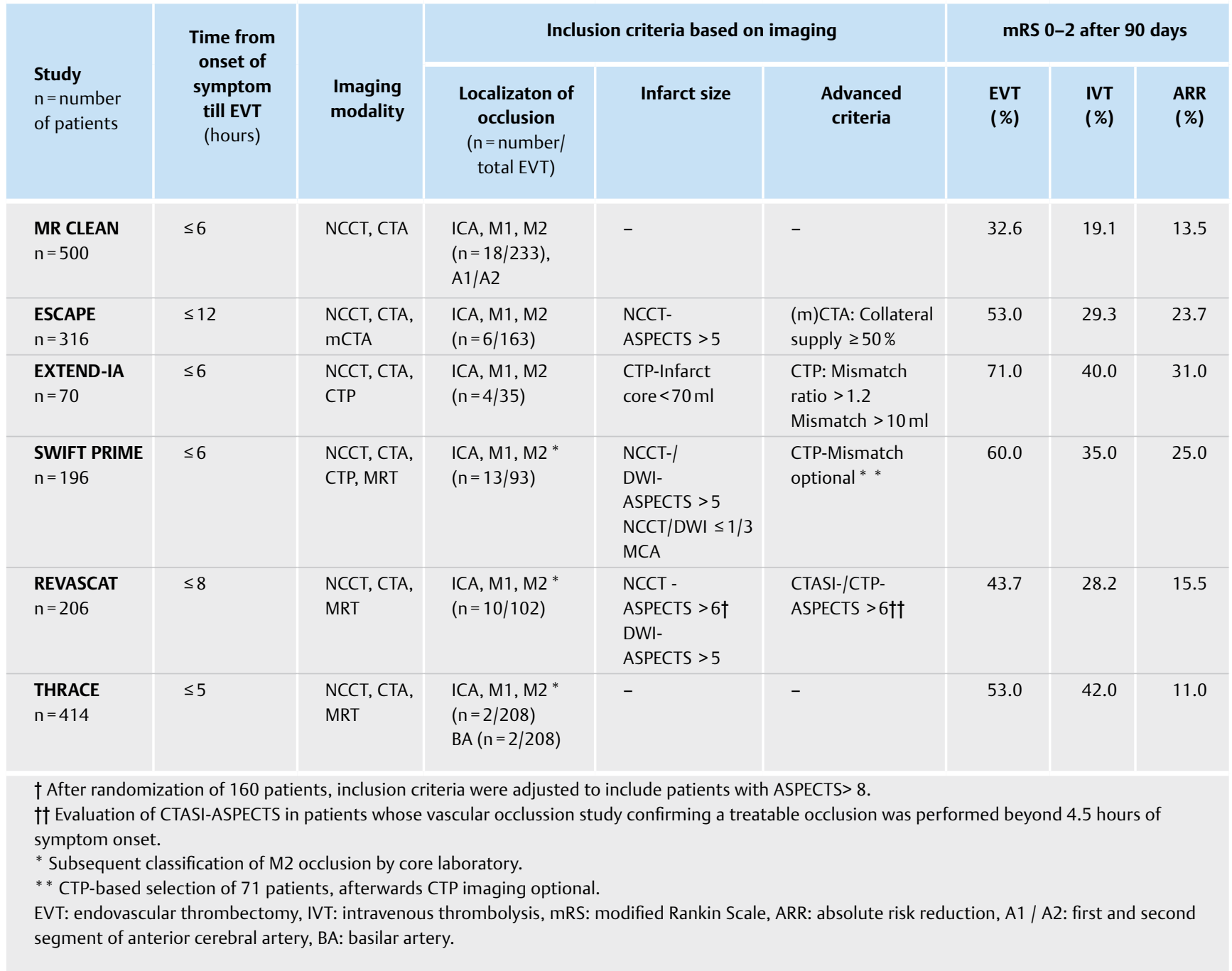

\section{Principles of Pretreatment Stroke Imaging}

Primary neuroimaging of acute stroke should be available anytime, anywhere. Imaging tools should be fast and reliable to perform and easy to interpret $[15,16]$. Ideally, imaging helps to identify all patients who will benefit from endovascular therapy and to exclude those who will not benefit or who may even be harmed by it. The basic information from non-invasive neuroimaging to evaluate endovascular thrombectomy should comprise: 1) exclusion of intracranial hemorrhage; 2 ) confirmation and localization of a treatable target vessel occlusion; 3) detection of irreversible damaged ischemic brain tissue; 4) characterization of salvageable tissue; 5) imaging of brain-supplying arteries for intervention planning.

The "Time is Brain" concept stands against the time-consuming and mostly sequential generation, processing and interpretation of image information. An estimated 1.9 million neurons get lost each minute in which stroke is untreated [17]. In IMS III and ESCAPE, the probability of achieving a functionally independent outcome decreased by $12-15 \%$ and $8.3 \%$, respectively, every 30 -minute increase in time-to-reperfusion $[18,19]$. In a stepwise diagnostic approach, it should therefore be clear before each further step whether the existing informations reliably indicate or contraindicate thrombectomy, and whether additional imaging studies will support decision-making or just delay initiation of treatment [20].

\section{Noncontrast Computed Tomography}

Computed tomography (CT) is commonly used for primary diagnosis in patients with acute stroke. It is widely available and can be carried out easily and fast. Noncontrast CT (NCCT) enables reliable exclusion of intracranial hemorrhage. Local hypoattenuation and swelling of brain parenchyma are early indicators of ischemic changes [21]. Detection of these early signs of cerebral infaction within $6 \mathrm{~h}$ of onset of symptoms is highly specific for irreversible ischemic brain damage, the extent of which is a decisive determinant for the clinical outcome after recanalization therapy [22, 23]. All positive randomized controlled thrombectomy studies have used information from NCCT for patient selection. 


\section{ASPECTS}

The Alberta Stroke Program Early CT Score (ASPECTS) is a easy-touse and widely established score to systematically quantify the extent of early ischemic changes in the anterior circulation on NCCT. The MCA territory is evaluated on two standardized levels and a total of 10 regions. For detection of early ischemic changes, a point on a scale of $0-10$ is deducted per defined region ( $\triangleright$ Fig. 1,3 ) $[24,25]$.

The retrospective analysis of the PROACT II data already indicated that patients with a small infarct core (ASPECTS > 7) could benefit from endovascular therapy, whereas no benefit could be demonstrated for patients with extensive infarct core (ASPECTS 57 ) [26]. ASPECTS was a strong predictor of clinical outcome and reperfusion after thrombectomy in IMS III [27]. In a joint analysis of the Penumbra Pivotal and Penumbra Imaging Collaborative studies of 2014 , only $5 \%(n=2 / 40)$ of patients with an initial ASPECTS of $0-4$ had a favourable clinical outcome after thrombectomy treatment and the mortality rate was $55 \%(n=22 / 40)$ [28]. MR CLEAN did not define inclusion criteria based on ASPECTS. However, subsequent analysis of the CT data revealed a median ASPECTS of 9 (interquartile range (IQR) 7-10), and a subgroup analysis showed that there was no significant treatment effect for an ASPECTS < 5 (adjusted odds ratio (OR): 1.09; $95 \%$ confidence interval (Cl): 0.14-8.46; $n=28$ patients) [1]. With ASPECTS $>5$ in ESCAPE and SWIFT PRIME as well as ASPECTS $>6$ in REVASCAT, a threshold was defined in each of these trials to exclude patients with extensive early ischemic changes. Subgroup analyses demonstrated no significant change of positive treatment effect comparing size of infarction above these ASPECTS thresholds [2, 4, 5]. This was confirmed in a recent meta-analysis of the 5 randomized controlled trials of 2015. Patients with small (ASPECTS 9-10) and moderate (ASPECTS 6-8) infarct size had a similar positive treatment effect (OR: 2.66; $95 \% \mathrm{Cl}: 1.61-4.40$ and OR: 2.34; $95 \% \mathrm{Cl}$ : 1.68-3.26) [29].

However, the meta-analysis of $n=121$ randomized patients with ASPECTS 0-5 in these studies could not confirm a significant treatment effect of thrombectomy (OR: 1.24; $95 \%$ Cl: 0.62-2.49) [29]. THRACE, like MR CLEAN, retrospectively analyzed the initial ASPECTS. Interestingly, approximately $30 \%(n=17 / 57)$ of the randomized patients with ASPECTS $0-4$ had a favourable clinical outcome [6]. Further trials are needed to specifically investigate this patient subgroup.

\section{Hyperdense Artery Sign}

The phenomenon of a thrombus that can be seen to be denser in comparison to circulating blood and the surrounding soft tissue on NCCT is described as the "hyperdense artery sign" [21]. The hyperdense artery sign is highly specific but not very sensitive to vessel occlusion of the middle cerebral artery [30,31]. Calcified plaques and high hematocrit levels also lead to vascular hyperdensities that can mimic a hyperdense artery sign. In the recently published randomized controlled thrombectomy study THERAPY [32], the detection of a $\geq 8 \mathrm{~mm}$-long hyperdense artery sign, which was confirmed by an additional CT angiography as a sign of vessel occlusion, was defined as an inclusion criterion. However, this study was terminated prematurely after the publication of MR CLEAN and did not achieve its primary endpoints with an underpowered sample. None of the 6 positive randomized controlled trials considered the hyperdense artery sign as a selection criterion.

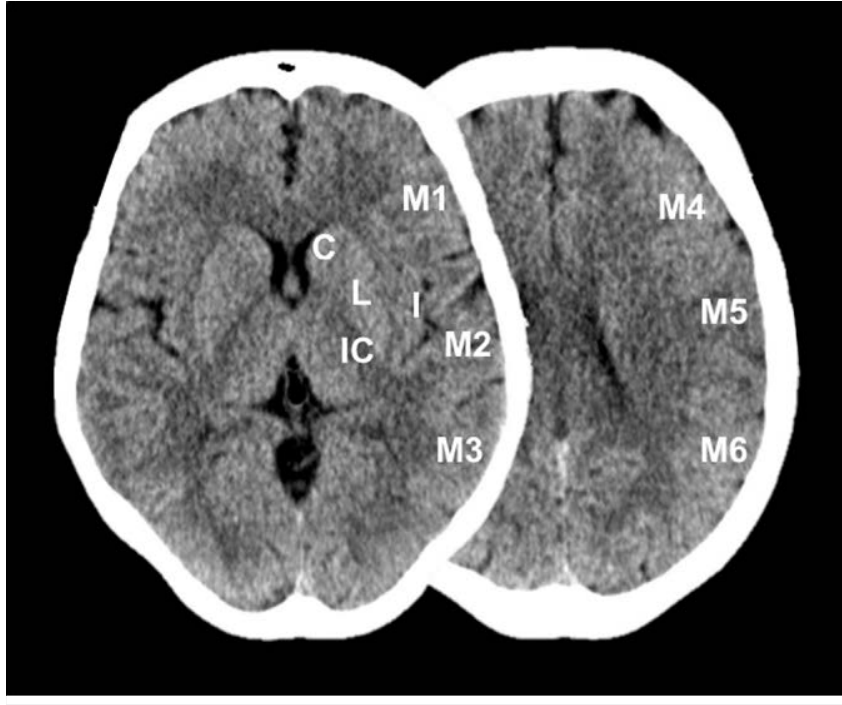

-Fig. 1 Alberta Stroke Program Early CT Score. Nonenhanced computed tomography with the 10 regions of the Alberta Stroke Program Early CT Score (ASPECTS) in the ganglionic and supraganglionic level of the MCA territory [24, 25]. C: caudate nucleus, L: lentiform nucleus, IC: internal capsule, I: insula; M1-M6: Cortex regions of the MCA territory.

\section{Computed Tomography Angiography}

Computed tomography angiography (CTA) with contrast-enhanced imaging of the cerebral blood vessels is quick to perform and has high sensitivity and specificity for the detection of intracranial artery occlusion [33]. In IMS III with overall neutral results, a post hoc analysis indicated that thrombectomy in patients with proximal intracranial artery occlusion detected by CTA could lead to higher recanalization rates and a better clinical outcome compared to intravenous thrombolysis alone [34].

\section{Localization of Vascular Occlusion}

All positive thrombectomy studies used CTA to identify patients with a treatable target vessel occlusion in the intracranial carotid artery (ICA) and the main trunk (M1 segment) of the middle cerebral artery (MCA). The results of the trials clearly confirm that patients with an occlusion in these intracranial arterial segments significantly benefit from endovascular thrombectomy [29].

\section{M2 Occlusion}

In the trials, only a few patients with an isolated occlusion in the $\mathrm{M} 2$ segment of the MCA were randomized ( $\triangleright$ Table 1 ). Interestingly, in SWIFT PRIME, REVASCAT and THRACE these occlusions were primary classified as $\mathrm{M} 1$ and only subsequent as $\mathrm{M} 2$ by the respective core laboratory [4-6]. The meta-analysis of the 5 positive studies of 2015, which comprised a total of $n=94$ patients with an $M 2$ occlusion, could not demonstrate a significant treatment effect of thrombectomy (OR: 1.28; $95 \% \mathrm{Cl}: 0.51-3.21$ ) [29].

\section{Tandem Occlusion}

A meta-analysis of the positive trials involved a total of $n=122$ patients who had an ipsilateral occlusion of the extracranial ICA in addition to intracranial occlusion. These patients with so-called "tan- 


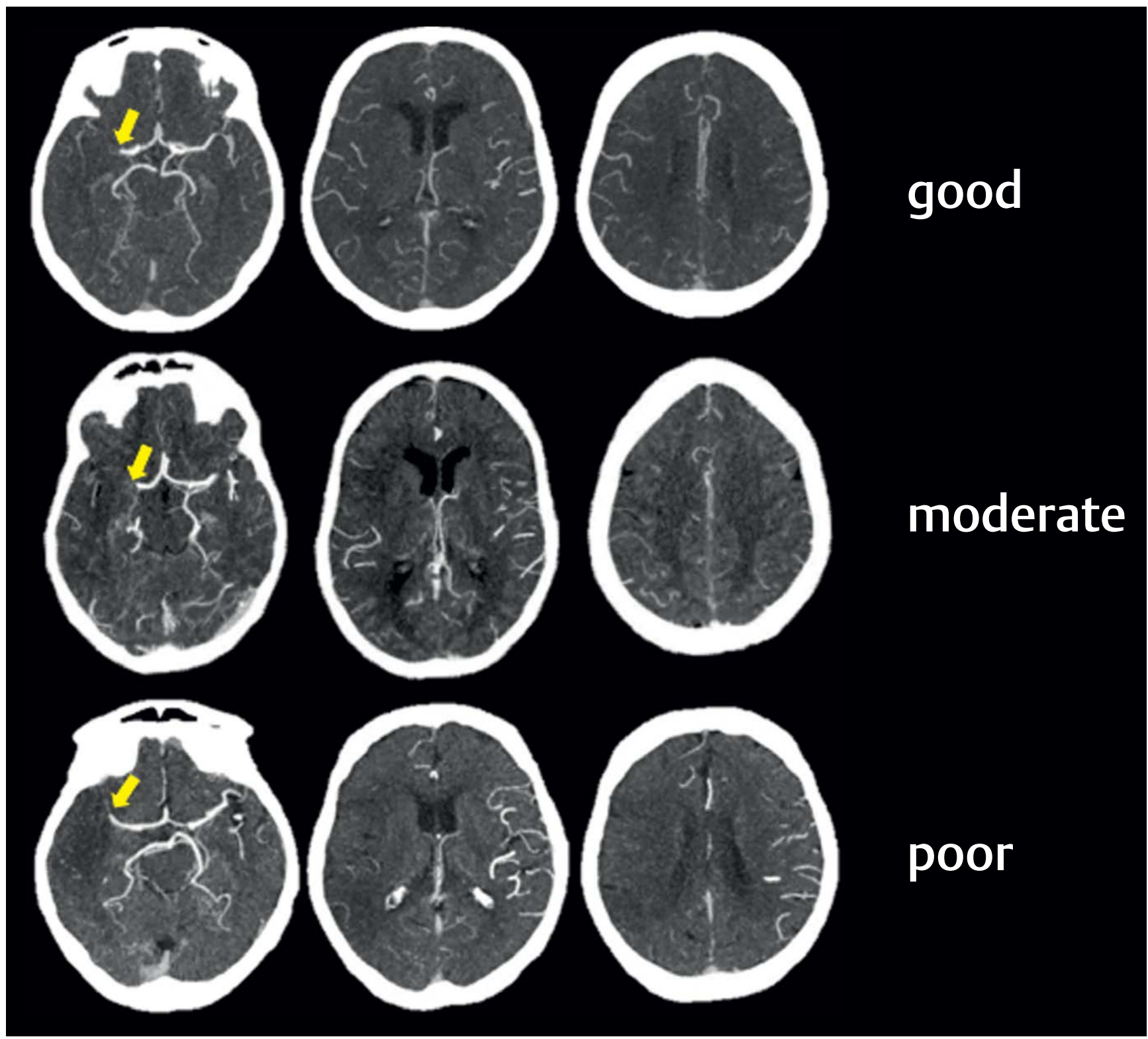

> Fig. 2 Collateral status. Computed tomographic angiography in a maximum intensity projection (MIP) of 3 patients with visualization of the collaterals in the right MCA territory with occlusion of the main trunk (yellow arrow). The first patient (top) has a good (collateral filling as in the non-affected hemisphere), the second patient has moderate and the third patient poor collateral blood flow (collaterals $>50 \%$ or $<50 \%$ compared to the unaffected hemisphere) [46].

dem occlusion" benefited equally from thrombectomy as patients $(n=1132)$ with an isolated intracranial occlusion (OR: $2.95 ; 95 \% \mathrm{Cl}$ : 1.38-6.32 and OR: 2.35 ; $95 \% \mathrm{Cl}: 1.68-3.28$, p-value $=0.17)$. The results indicate that these patients should not be excluded from treatment [29]. It is currently unclear whether the acute intervention should include, in addition to the recanalization of the intracranial artery occlusion, a stent-protected angioplasty of the ICA.

\section{CTA and Intervention Planning}

The CTA provides important information for the planning and preparation of the endovascular procedure. Knowledge about the individual anatomy and pathology of the aortic arch, neck vessels and the target vessel allow the interventionist to choose the appropriate treatment strategy. Moreover, detailed pretreatment CTA-imaging of these vascular structures can reduce the duration of endovascular procedure [35, 36].

\section{"tissue window"}

In addition to direct imaging of the vascular system, CTA can also provide important indirect information on the status of the brain tissue. In the source images of the CTA (CTASI), a decreased enhancement of the brain parenchyma is a surrogate for reduced cerebral blood volume. The CTASI are more sensitive in detection of early ischemic changes compared to NCCT [37, 38]. In REVASCAT, a CTASI-ASPECTS was added to NCCT in patients whose vascular occlus- 
sion study confirming a treatable occlusion was performed beyond 4.5 hours of symptom onset [5]. On this issue no results have been published so far. Quantification of the infarct core depends on CTA acquisition protocol. Fast image acquisition and decreased injection flow rates of contrast medium can lead to an overestimation of the infarct size in the CTASI [39].

\section{Collateral Status}

The size and temporal progression of cerebral infarction after arterial occlusion are dependent on the arterial collateral supply, which is highly variable between individuals [40]. A good leptomeningeal collateral flow in pretreatment imaging is associated with a higher probability for a good clinical outcome, lower mortality and a lower risk of hemorrhage in stroke patients treated with endovascular thrombectomy [41].

In the CTA, the grade of leptomeningeal collateral flow can be estimated and quantified using the ratio of retrograde pial arterial filling distal to vascular occlusion compared to the contralateral asymptomatic hemisphere [42] ( Fig. 2). In ESCAPE, in addition to NCCT, a CTA-based visualization of collateral flow was used for assessing the brain tissue status. Patients who had no or only minimal collateral filling in more than $50 \%$ of the affected MCA territory compared to the contralateral hemisphere were not included in the study [2].

However, determining the collateral status in the conventional single-phase CTA has limitations. When filling of the pial arteries distal to the occlusion is detected too early in a single frame image acquisition, the actual grade of collaterals is underestimated. A dynamic CTA, which can be obtained, for example, from the data sets of CT perfusion (CTP), offers better visualization of the actual extent of collaterals. Moreover, results of dynamic CTA have higher correlation to imaging and clinical outcome $[43,44]$. In ESCAPE, the majority of patients were examined with a multiphase $(m)$ CTA. This technique allows fast and time-resolved assessment of the collaterals. Compared to the dynamic CTA from CTP data sets, MCTA requires lower radiation dose and no additional administration of contrast agent[45].

The positive results of ESCAPE indicate that patients, who will probably benefit from endovascular treatment 6-12 $\mathrm{h}$ after symptom onset, can be identified by the combination of ASPECTS and CTA collaterals [2]. A recent post-hoc analysis of MR CLEAN confirmed the high prognostic value of collateral status for clinical outcome. Pretreatment CTA collateral status significantly modified treatment effect of endovascular thrombectomy ( $p$-value $=0.038$ ). The benefit of thrombectomy was greatest in patients with good collateral flow (OR: 3.2; $95 \% \mathrm{Cl}$ : 1.7-6.2), whereas treatment benefit was less or not detectable in patients with poor or absent collateral flow (OR: 1.2; $95 \% \mathrm{Cl}: 0.7-2.3$ and OR: 1.0; $95 \% \mathrm{Cl}: 0.1-8.7$ ) [46].

\section{Computed Tomography Perfusion}

Computed tomographic perfusion (CTP) is a dynamic contrast-enhanced imaging technique, from which various parameters can be calculated that represent the current perfusion status of the brain tissue. Experimental investigations have shown that in acute ischemic stroke, the infarct core can be distinguished from a adjoining critically hypoperfused penumbra. This endangered brain tissue can be potentially recovered by early reperfusion. Without reperfusion, the infarct core expands into the area of the penumbra depending on duration and severity of reduced perfusion [47].

Modern CT scanners and evaluation algorithms allow a fast whole-brain perfusion imaging with largely automated, user-independent identification and quantification of the infarct core and penumbra $[48,49]$. There is, however, no consensus as to which perfusion parameters and threshold values are most suitable for the depiction of the tissue status [50, 51]. EXTEND-IA and SWIFT PRIME used a CTP with automated data processing [3, 4] for patient selection. The applied software (RAPID, iSchemaView, Inc., Redwood City, California, USA) calculates the infarct core using a relative cerebral blood flow ( $\mathrm{rCBF}$ ) $<30 \%$ compared to the contralateral hemisphere and uses a time to maximum (Tmax) $>6$ s to visualize the penumbra ( $\triangleright$ Fig. 3 ). Both parameters and the respective thresholds were assessed as reliable surrogate markers in various studies [52-56]. EXTEND-IA selected study participants on the basis of CTP and defined the inclusion criteria for the infarct core as < 70 milliliters $(\mathrm{ml})$, a mismatch ratio (penumbra/infarct core) $>1.2$ and an absolute mismatch volume (penumbra - infarct core) $>10 \mathrm{ml}$. The acquisition, evaluation and interpretation of these data took less than $15 \mathrm{~min}$. The results show that automated CTP imaging is applicable to asses patients with acute stroke, in particular to evaluate them for endovascular thrombectomy. It can be performed quickly and identifies patients who greatly benefit from endovascular therapy compared to intravenous thrombolysis alone ( Table 1) $[3,49]$.

In MR CLEAN, an optional CTP was performed in $66.8 \%$ $(n=334 / 500)$ of the study patients before randomization. Subsequent evaluation of $n=175$ available CTP data sets confirmed that an extensive infarct core is associated with poor clinical outcome. However, the CTP parameters for infarct core, penumbra or mismatch did not have any additional impact on the treatment effect of thrombectomy. CTP selection would have excluded patients from endovascular treatment who had in fact benefited from therapy [57]. Finally, the further advantage of adding CTP for guiding treatment decision on endovascular thrombectomy in these preselected study groups remains open. However, it is clear that the achievement of a higher treatment effect by application of advanced inclusion criteria is likely to exclude patients who might benefit from the therapy [3].

\section{Magnetic Resonance Imaging}

With diffusion-weighted imaging (DWI), magnetic resonance imaging (MRI) allows for highly specific and sensitive detection of ischemic changes within the first $6 \mathrm{~h}$ after onset of symptoms [58]. A multiparametric stroke protocol consisting of DWI, fluid attenuated inversion recovery (FLAIR) or T2-weighted sequence, bloodsensitive sequence (susceptibility-weighted imaging (SWI) or T2*-weighted sequence), vascular imaging (contrast-enhanced, time-of- flight or phase-contrast angiography) and perfusion imaging (PWI) can provide the relevant information for endovascular thrombectomy evaluation. In fact, examination times of less than 10 min can be achieved [58,59]. Significant limitations of MRI in acute stroke imaging include limited availability, costly examination preparation and patient monitoring as well as contraindications for various implants, such as cardiac pacemakers and high susceptibility to movement artefacts $[16,60]$. 


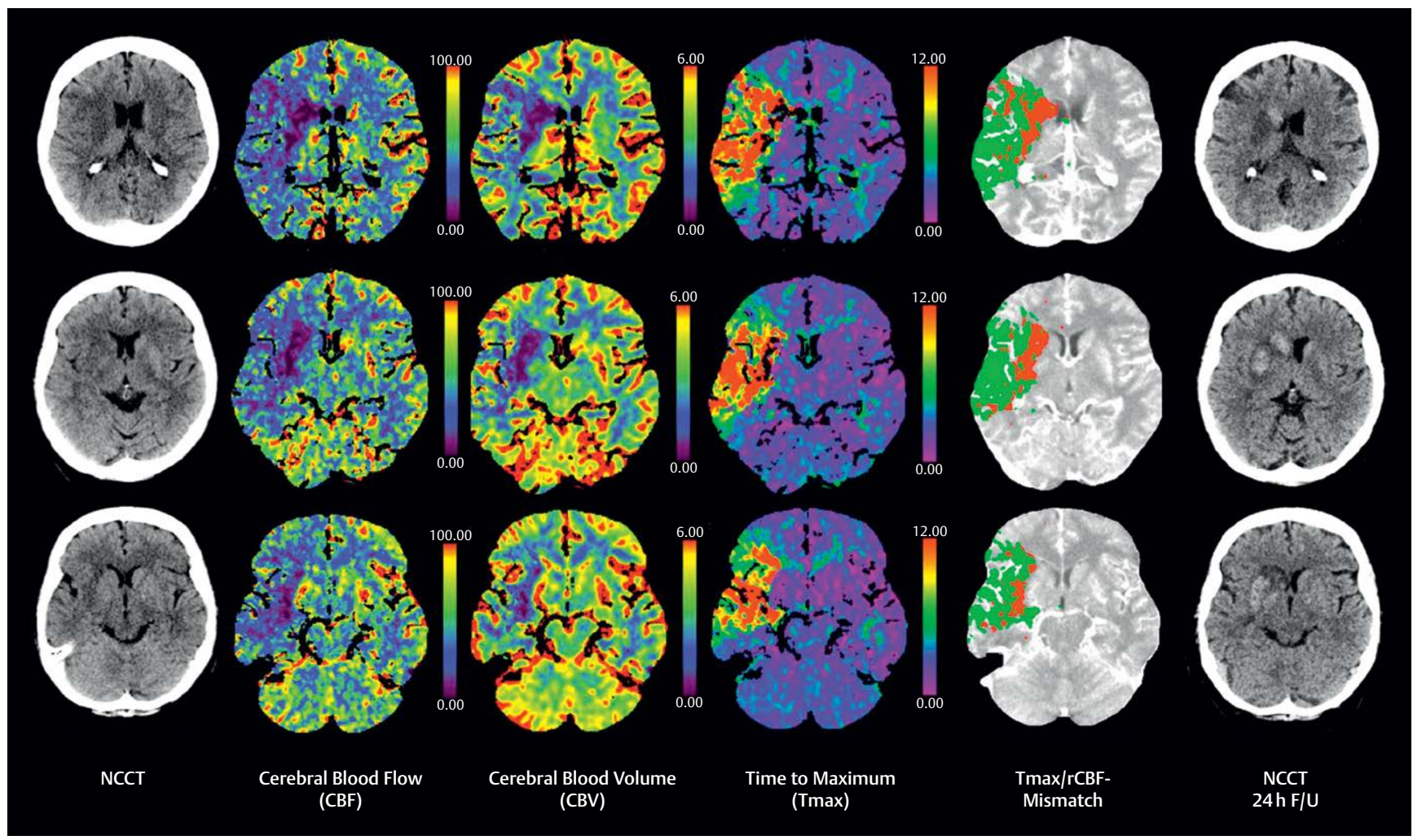

- Fig. 3 Imaging of infarct core and penumbra. Noncontrast computed tomography (NCCT) and parameter maps of a computed tomographic perfusion (CTP) of a patient with an acute ischemic stroke in the right MCA territory an the NCCT follow-up $24 \mathrm{~h}$ after thrombectomy (24h $\mathrm{F} / \mathrm{U}$ ). In NCCT, hypoattenuation can be found in the caudate nucleus, lentiform nucleus, insula and in the cortex region M4 of the right MCA territory (ASPECTS 6). The parameter maps of CBF and Tmax indicate a large area of hypoperfusion in the right MCA territory with involvement of the basal ganglia. The CBV parameter map indicates a hypoperfusion area comparable to NCCT findings. The Tmax/rCBF mismatch visualises the infarct core $(\mathrm{rCBF}<30 \%)$ in red and the penumbra $(\mathrm{Tmax}>6 \mathrm{~s})$ in green. In the NCCT $24 \mathrm{~h}$ F/U after successful thrombectomy, infarction with partial hemorrhagic transformation is shown. The final infarct size corresponds to the infarct core on CTP.

SWIFT PRIME and REVASCAT also selected MRI-based inclusion criteria and acquired MRI data sets of $17.4 \%(n=34 / 195)$ and $5.3 \%$ ( $n=11 / 206)$ of randomized patients $[4,5,12]$. However, no meaningful information can be derived from these subgroups due to the small number of patients. The randomized controlled trial MR RESCUE, which included the majority of patients based on MRI perfusion imaging ( $80 \%, n=94 / 118)$, did not show a benefical effect of endovascular thrombectomy, but the rate of recanalization was very low [10]. Despite the limited study situation, MRI, if performed without substantial delay, seems suitable for adequate evaluation of patients before thrombectomy. Equivalence or superiority to imaging with the better-studied NCCT and CTA has not yet been scientifically investigated.

\section{Imaging and Extended Time Window}

The average time from symptom onset to the start of thrombectomy (time of groin puncture) was 285 min (standard deviation: 210$362 \mathrm{~min}$ ) in the 5 positive studies of 2015, with suitable patients in MR CLEAN, SWIFT PRIME and EXTEND-IA being included up to $6 \mathrm{~h}$ after onset of symptoms [1, 3, 4, 29]. REVASCAT randomized $9.7 \%$ $(n=20 / 206)$ of the study participants in an extended time window of $6-8 \mathrm{~h}$, but did not publish an independent analysis of this small group of patients [5]. In ESCAPE, 15.5\% ( $n=49 / 315)$ of the patients were included in the $6-12 \mathrm{~h}$ time window based on the combination of ASPECTS and CTA collateral status. Subgroup analysis indicated a trend in favor of thrombectomy (RR: 1.7; $95 \% \mathrm{Cl}: 0.7-4.0$ ) [2]. The meta-analysis of $n=208$ patients from the trials published in 2015 , with a time window $>5 \mathrm{~h}$ from symptom onset to randomization, also indicated that selected patients can benefit from thrombectomy (OR: 1.76; 95\% Cl: 1.05-2.97) [29]. Further evidence of a possible beneficial effect of thrombectomy in the extended time window was provided by the subsequent analyses of DEFUSE 2 [61]: In patients with a mismatch profile in perfusion imaging, the relationship between endovascular reperfusion and good clinical outcome was not time-dependent [62] and patients in a time window $>6 \mathrm{~h}$ may benefit from thrombectomy [63].

\section{Indicators for Complications of Thrombectomy}

Overall, the 6 positive thrombectomy studies reported low complication rates and a good safety profile of the thrombectomy devices used [1-6]. Periprocedural complications of mechanical recanalization of intracranial artery occlusions that may result in increased morbidity and mortality include intracranial hemorrhage, embolism in adjoining vessel territories, arterial wall damage and 
vasospasm $[64,65]$. The identification and evaluation of risk factors for these complications in pretreatment imaging could help to identify patients who might be harmed by thrombectomy. In fact, there are indicators, for instance, of a correlation between the collateral status and parameters of the CTP, on the one hand, and the risk of intracranial hemorrhage, on the other $[41,66]$. Further studies are needed to assess the role of imaging-based predictors for complications of mostly multifactorial origin and to establish an individual risk stratification for thrombectomy.

\section{Conclusion and Outlook}

The recently published randomized controlled trials confirm that selected patients with a proximal anterior circulation artery occlusion (ICA, M1) and small infarct core benefit from endovascular thrombectomy in a time window up to $6 \mathrm{~h}$ after onset of symptoms. These patients should be immediately identified with non-invasive neuroimaging including imaging of the vascular system and treated at appropriate centers. In addition to obligatory detection of a treatable target vessel occlusion and exclusion of other causes for acute neurological symptoms, different imaging-based approaches can be used in order to select patients who are likely to benefit from endovascular therapy with great probability. The current trials were primarily designed to evaluate the therapy strategy. Which imaging strategy is most suitable for guiding treatment decision on endovascular thrombectomy cannot be conclusively assessed on the basis of the available data. Studies such as SELECT (ClinicalTrials.gov Identifier: NCT02446587) or PRACTISE (ClinicalTrials.gov Identifier: NCT02360670) are currently recruiting study participants to answer this question.

Obviously, most patients in the positive trials were evaluated for endovascular treatment on the basis of CT imaging. MR CLEAN and THRACE, the studies with the least selective inclusion criteria in pretreatment imaging ( $\triangleright$ Table $\mathbf{1}$ ), have already demonstrated a clear treatment effect for thrombectomy, with the majority of the patients having a small infarct core. A good clinical outcome after thrombectomy was associated with a small infarct core in the positive studies. The size of early ischemic changes can be estimated on NCCT (e. g., ASPECTS), with DWI, via perfusion imaging with automated processing (e. g., rCBF <30\%) or indirectly via CTA (e. g., CTASI). However, each of these methods has limitations with regard to diagnostic safety.

Further studies are needed to clarify if patients with an extensive infarct core (e. g., ASPECTS $\leq 5$, absolute volume $>70 \mathrm{ml}$ ) also benefit from thrombectomy and why some patients do not have a good clinical outcome after rapid recanalization despite small initial infarct core. Additional perfusion imaging or visualization of collaterals could play an important role in these patient groups.

Perfusion imaging and a combination of ASPECTS and CTA collateral status also seem to be able to identify patients who may benefit from endovascular thrombectomy in an extended time win- dow ( $>6 \mathrm{~h}$ ) or in case of unclear symptom onset. Trials such as DAWN (ClinicalTrials.gov Identifier: NCT02142283) or DEFUSE 3 (ClinicalTrials.gov Identifier: NCT02586415) that, in a time window of 6-24h, or 6-16 h, respectively, after onset of symptoms, randomize patients for thrombectomy treatment based on perfusion imaging will answer these questions.

The value of thrombectomy in M2 occlusion is unclear. Mechanical recanalization of $\mathrm{M} 2$ occlusions appears to be feasible in selected patients and has a good safety profile [67]. Currently, no evidence-based recommandations can be given.

The positive results of recent thrombectomy trials are based on the endovascular treatment of arterial occlusion in the anterior circulation and ultimately do not allow any final conclusions on patients with an intracranial occlusion in the vertebrobasilar circulation. The ENDOSTROKE registry reported high recanalization rates for thrombectomy in patients with basilar artery occlusion [68]. Finally, the results of BASICS [69] (ClinicalTrials.gov Identifier: NCT01717755) and other randomized controlled trials are needed to clarify the role of thrombectomy in the posterior circulation as well as the benefits of pretreatment imaging on selection of suitable patients.

\section{Practical Conclusions}

Based on the current study situation and guidelines [13], the following recommendations for neuroimaging in guiding treatment decision on endovascular thrombectomy arise:

- Patients with acute stroke should immediately receive non-invasive neuroimaging.

- In ischemic stroke, vascular imaging (CTA, MRA) should be performed immediately to select patients for thrombectomy.

- Thrombectomy is recommended for patients with intracranial large-vessel occlusion (ICA, M1, restricted for $M 2$ ) and imaging signs of a small infarct core (e. g., ASPECTS > 5) in a time window of up to $6 \mathrm{~h}$ after onset of symptoms.

- Patients in an extended time window (>6h) or with extensive infarction (e.g., ASPECTS $\leq 5$ ) should not, as a matter of principle, be excluded from thrombectomy. Advanced imaging (perfusion, collaterals) can be used to select patients with salvageable brain tissue.

- Patients with acute basilar artery occlusion should be treated primarily with endovascular thrombectomy or included in randomized trials.

\section{Conflict of interest}

The Dresdner University Stroke Center (DUSC) has participated in the studies IMS III and THERAPY and is currently participating in the BASICS study. The authors state that there is no conflict of interest. 


\section{LIST OF ABBREVIATIONS}

\begin{tabular}{|c|c|}
\hline BASICS & $\begin{array}{l}\text { Basilar Artery International Coopera- } \\
\text { tion Study }\end{array}$ \\
\hline DAWN & $\begin{array}{l}\text { Trevo and Medical Management vs. } \\
\text { Medical Management Alone in Wake } \\
\text { Up and Late Presenting Strokes }\end{array}$ \\
\hline DEFUSE 2 & $\begin{array}{l}\text { Diffusion Weighted Imaging Evalua- } \\
\text { tion for Understanding Stroke } \\
\text { Evolution Study } 2\end{array}$ \\
\hline DEFUSE 3 & $\begin{array}{l}\text { Endovascular Therapy Following Imaging } \\
\text { Evaluation for Ischemic Stroke } 3\end{array}$ \\
\hline ENDOSTROKE & $\begin{array}{l}\text { International Multicenter Registry for } \\
\text { Mechanical Recanalization Procedures } \\
\text { in Acute Stroke }\end{array}$ \\
\hline ESCAPE & $\begin{array}{l}\text { Endovascular Treatment for Small } \\
\text { Core and Proximal Occlusion Ischemic } \\
\text { Stroke }\end{array}$ \\
\hline EXTEND-IA & $\begin{array}{l}\text { Extending the Time for Thrombolysis } \\
\text { in Emergency Neurological Deficits } \\
\text { - Intra-Arterial }\end{array}$ \\
\hline
\end{tabular}

IMS III Interventional Management of Stroke (IMS) III Trial

MR CLEAN A Multicenter Randomized Clinical Trial of Endovascular Treatment for Acute Ischemic Stroke in The Netherlands

MR RESCUE Mechanical Retrieval and Recanalization of Stroke Clots Using Embolectomy

\begin{tabular}{|c|c|}
\hline PRACTISE & $\begin{array}{l}\text { Penumbra and Recanalisation Acute } \\
\text { Computed Tomography in Ischaemic } \\
\text { Stroke Evaluation }\end{array}$ \\
\hline PROACT II & $\begin{array}{l}\text { Prolyse in Acute Cerebral Thrombo- } \\
\text { embolism II }\end{array}$ \\
\hline REVASCAT & $\begin{array}{l}\text { Endovascular Revascularization With } \\
\text { Solitaire Device vs. Best Medical } \\
\text { Therapy in Anterior Circulation Stroke } \\
\text { Within } 8 \mathrm{~h}\end{array}$ \\
\hline SELECT & $\begin{array}{l}\text { Optimizing Patient's Selection for } \\
\text { Endovascular Treatment in Acute } \\
\text { Ischemic Stroke }\end{array}$ \\
\hline SWIFT PRIME & $\begin{array}{l}\text { Solitaire With the Intention For } \\
\text { Thrombectomy as PRIMary Endovas- } \\
\text { cular Treatment }\end{array}$ \\
\hline $\begin{array}{l}\text { SYNTHESIS } \\
\text { EXP }\end{array}$ & $\begin{array}{l}\text { Intra-arterial vs. Systemic Thromboly- } \\
\text { sis for Acute Ischemic Stroke }\end{array}$ \\
\hline THERAPY & $\begin{array}{l}\text { Assess the Penumbra System in the } \\
\text { Treatment of Acute Stroke }\end{array}$ \\
\hline THRACE & $\begin{array}{l}\text { Trial and Cost Effectiveness Evaluation } \\
\text { of Intra-arterial Thrombectomy in } \\
\text { Acute Ischemic Stroke }\end{array}$ \\
\hline
\end{tabular}

[1] Berkhemer OA, Fransen PSS, Beumer D et al. A randomized trial of intraarterial treatment for acute ischemic stroke. N Engl J Med 2015; 372: $11-20$

[2] Goyal M, Demchuk AM, Menon BK et al. Randomized assessment of rapid endovascular treatment of ischemic stroke. N Engl J Med 2015; 372: 1019-1030

[3] Campbell BCV, Mitchell PJ, Kleinig T] et al. Endovascular therapy for ischemic stroke with perfusion-imaging selection. N Engl J Med 2015; 372: 1009-1018

[4] Saver JL, Goyal M, Bonafe A et al. Stent-retriever thrombectomy after intravenous t-PA vs. t-PA alone in stroke. N Engl J Med 2015; 372 : 2285-2295

[5] Jovin TG, Chamorro A, Cobo E et al. Thrombectomy within 8 hours after symptom onset in ischemic stroke. N Engl J Med 2015; 372: $1-11$

[6] Bracard S, Ducrocq X, Mas JL et al. Mechanical thrombectomy after intravenous alteplase versus alteplase alone after stroke (THRACE): a randomised controlled trial. Lancet Neurol 1995; 15: 1581-1587

[7] Grotta JC, Hacke W. Stroke Neurologist's Perspective on the New Endovascular Trials. Stroke 2015; 46: 1447-1452

[8] Goyal M, Menon BK. Variability of results of recent acute endovascular trials: a statistical analysis. J Neurointerv Surg 2016; 8: 875-877

[9] Broderick JP, Palesch YY, Demchuk AM et al. Endovascular therapy after intravenous t-PA versus t-PA alone for stroke. N Engl J Med 2013; 368 : 893-903

[10] Kidwell CS, Jahan R, Gornbein J et al. A trial of imaging selection and endovascular treatment for ischemic stroke. N Engl J Med 2013; 368: 914-923

[11] Ciccone A, Valvassori L, Nichelatti M et al. Endovascular treatment for acute ischemic stroke. N Engl J Med 2013; 368: 904-913

[12] Warach SJ, Luby M, Albers GW et al. Acute stroke imaging research roadmap III imaging selection and outcomes in acute stroke reperfusion clinical trials: consensus recommendations and further research priorities. Stroke 2016; 47: 1389-1398

[13] Ringleb PA, Hamann GF, Röther J et al. Akuttherapie des ischämischen Schlaganfalls - Rekanalisierende Therapie: Ergänzung 2015. Akt Neurol 2016; 43: 82-91

[14] Powers W], Derdeyn CP, Biller J et al. 2015; American Heart Association/American Stroke Association Focused Update of the 2013 Guidelines for the early management of patients with acute ischemic stroke regarding endovascular treatment: a guideline for healthcare professionals from the American. Stroke 2015; 46: 3020-3035

[15] Puetz V, Barlinn K, Bodechtel U et al. Imaging-based selection for revascularization in acute ischemic stroke. Curr Opin Neurol 2016; 29 : 20-29

[16] Menon BK, Campbell BCV, Levi C et al. Role of imaging in current acute ischemic stroke workflow for endovascular therapy. Stroke 2015; 46: 1453-1461

[17] Saver JL. Time is brain - Quantified. Stroke 2006; 37: 263-266

[18] Menon BK, Sajobi TT, Zhang Y et al. Analysis of workflow and time to treatment on thrombectomy outcome in the endovascular treatment for small core and proximal occlusion ischemic stroke (ESCAPE) randomized, controlled trial. Circulation 2016; 133: 2279-2286

[19] Khatri P, Yeatts SD, Mazighi M et al. Time to angiographic reperfusion and clinical outcome after acute ischaemic stroke: An analysis of data from the Interventional Management of Stroke (IMS III) phase 3 trial. Lancet Neurol 2014; 13: 567-574

[20] Goyal M, Fargen KM, Menon BK. Acute stroke, Bayes' theorem and the art and science of emergency decision-making. J Neurointerv Surg 2013; 6: 2-6 
[21] Von Kummer R, Meyding-Lamade U, Forsting M et al. Sensitivity and prognostic value of early $\mathrm{CT}$ in occlusion of the middle cerebral artery trunk. AJNR 1994; 15: 9-18

[22] Jovin TG, Yonas H, Gebel JM et al. The cortical ischemic core and not the consistently present penumbra is a determinant of clinical outcome in acute middle cerebral artery occlusion. Stroke 2003; 34 : 2426-2433

[23] von Kummer R, Bourquain H, Bastianello $\mathrm{S}$ et al. Early prediction of irreversible brain damage after ischemic stroke at CT. Radiology 2001; 219: $95-100$

[24] Puetz V, Dzialowski I, Hill MD et al. The Alberta stroke program early ct score in clinical practice: What have we learned? Int J Stroke 2009; 4: 354-364

[25] Barber PA, Demchuk AM, Zhang J et al. Validity and reliability of a quantitative computed tomography score in predicting outcome of hyperacute stroke before thrombolytic therapy. ASPECTS Study Group. Alberta Stroke Programme Early CT Score. Lancet 2000; 355: 1670-1674

[26] Hill MD, Rowley HA, Adler F et al. Selection of acute ischemic stroke patients for intra-arterial thrombolysis with pro-urokinase by using ASPECTS. Stroke 2003; 34: 1925-1931

[27] Hill MD, Demchuk AM, Goyal M et al. Alberta stroke program early computed tomography score to select patients for endovascular treatment interventional management of stroke (IMS)-III trial. Stroke 2014; 45: 444-449

[28] Yoo AJ, Zaidat OO, Chaudhry ZA et al. Impact of pretreatment noncontrast CT Alberta stroke program early ct score on clinical outcome after intra arterial stroke therapy. Stroke 2014; 45: 746-751

[29] Goyal M, Menon BK, Van Zwam WH et al. Endovascular thrombectomy after large-vessel ischaemic stroke: A meta-analysis of individual patient data from five randomised trials. Lancet 2016; 387: 1723-1731

[30] Wolpert SM, Bruckmann H, Greenlee R et al. Neuroradiologic evaluation of patients with acute stroke treated with recombinant tissue plasminogen activator. The rt-PA Acute Stroke Study Group. AJNR 1990; 14: 3-13

[31] Barber PA, Demchuk AM, Hill MD et al. The probability of middle cerebral artery MRA flow signal abnormality with quantified CT ischaemic change: targets for future therapeutic studies. J Neurol Neurosurg Psychiatry 2004; 75: 1426-1430

[32] Mocco ], Zaidat OO, Von Kummer R et al. Aspiration thrombectomy after intravenous alteplase versus intravenous alteplase alone. Stroke 2016; 47: 2331-2338

[33] Bash S, Villablanca JP, Jahan R et al. Intracranial vascular stenosis and occlusive disease: Evaluation with CT angiography, MR angiography, and digital subtraction angiography. AJNR 2005; 26: 1012-1021

[34] Demchuk AM, Goyal M, Yeatts SD et al. Recanalization and clinical outcome of occlusion sites at baseline CT angiography in the Interventional Management of Stroke III trial. Radiology 2014; 273: 202-210

[35] Almekhlafi M, Menon B, Goyal M. Lessons learnt from recent endovascular stroke trials: Finding a way to move forward. Exp Rev Cardiovasc Ther 2014; 12: 429-436

[36] Appireddy RMR, Demchuk AM, Goyal M et al. Endovascular therapy for ischemic stroke. J Clin Neurol 2015; 11: 1-8

[37] Bhatia R, Bal SS, Shobha $N$ et al. CT angiographic source images predict outcome and final infarct volume better than noncontrast CT in proximal vascular occlusions. Stroke 2011; 42: 1575-1580

[38] Coutts SB, Lev MH, Eliasziw M et al. ASPECTS on CTA source images versus unenhanced $C T$ : Added value in predicting final infarct extent and clinical outcome. Stroke 2004; 2472-2476

[39] Pulli B, Schaefer PW, Hakimelahi R et al. Acute ischemic stroke: infarct core estimation on CT angiography source images depends on CT angiography protocol. Radiology 2012; 262: 593-604
[40] Liebeskind DS. Collateral circulation. Stroke 2003; 34: 2279-2284

[41] Leng X, Fang H, Leung TWH et al. Impact of collaterals on the efficacy and safety of endovascular treatment in acute ischaemic stroke: a systematic review and meta-analysis. J Neurol Neurosurg Psychiatry 2015; 87: 1-8

[42] McVerry F, Liebeskind DS, Muir KW. Systematic Review of Methods for Assessing Leptomeningeal Collateral Flow. AJNR 2012; 33: 576-582

[43] Van Den Wijngaard IR, Holswilder G, Wermer MJH et al. Assessment of collateral status by dynamic ct angiography in acute mca stroke: Timing of acquisition and relationship with final infarct volume. AJNR 2016; 37: 1231-1236

[44] Frölich AM], Wolff SL, Psychogios MN et al. Time-resolved assessment of collateral flow using 4D CT angiography in large-vessel occlusion stroke. Eur Radiol 2014; 24: 390-396

[45] Menon BK, d'Esterre CD, Qazi EM et al. Multiphase CT angiography: a new tool for the imaging triage of patients with acute ischemic stroke Radiology 2015; 275: 510-520

[46] Berkhemer OA, Jansen IGH, Beumer D et al. Collateral status on baseline computed tomographic angiography and intra-arterial treatment effect in patients with proximal anterior circulation stroke. Stroke 2016; 47: 768-776

[47] Astrup ], Siesjö BK, Symon L. Thresholds in cerebral ischemia - the ischemic penumbra. Stroke 1981; 12: 723-725

[48] Bivard A, Levi C, Spratt N et al. Perfusion CT in acute stroke: a comprehensive analysis of infarct and penumbra. Radiology 2013; 267: 543-550

[49] Campbell BCV, Yassi N, Ma H et al. Imaging selection in ischemic stroke: Feasibility of automated CT-perfusion analysis. Int J Stroke 2015; 10: 51-54

[50] Dani KA, Thomas RGR, Chappell FM et al. Systematic review of perfusion imaging with computed tomography and magnetic resonance in acute ischemic stroke : heterogeneity of acquisition and stroke imaging study. Stroke 2012; 43: 563-566

[51] Heit J], Wintermark M. Perfusion computed tomography for the evaluation of acute ischemic stroke. Stroke 2016; 47: 1153-1158

[52] Campbell BCV, Christensen S, Levi CR et al. Cerebral blood flow is the optimal CT perfusion parameter for assessing infarct core. Stroke 2011; 42: 3435-3440

[53] Austein F, Riedel C, Kerby T et al. Comparison of Perfusion CT Software to Predict the Final Infarct Volume after Thrombectomy. Stroke 2016; 47: 2311-2317

[54] Olivot J-M, Mlynash M, Thijs VN et al. Optimal Tmax threshold for predicting penumbral tissue in acute stroke. Stroke 2009; 40: 469-475

[55] Kamalian S, Kamalian S, Maas MB et al. CT cerebral blood flow maps optimally correlate with admission diffusion-weighted imaging in acute stroke but thresholds vary by postprocessing platform. Stroke 2011; 42: 1923-1928

[56] Campbell BCV, Christensen S, Levi CR et al. Comparison of computed tomography perfusion and magnetic resonance imaging perfusion-diffusion mismatch in ischemic stroke. Stroke 2012; 43: 2648-2653

[57] Borst J, Berkhemer OA, Roos YB et al. Value of computed tomographic perfusion-based patient selection for intra-arterial acute ischemic stroke treatment. Stroke 2015; 46: 3375-3382

[58] Muir KW, Buchan A, von Kummer R et al. Imaging of acute stroke. Lancet Neurol 2006; 5: 755-768

[59] Nael K, Khan R, Choudhary G et al. Six-minute magnetic resonance imaging protocol for evaluation of acute ischemic stroke: Pushing the boundaries. Stroke 2014; 45: 1985-1991

[60] Wintermark M, Fiehler ], Kudo K et al. International survey of acute stroke imaging capabilities. We need you! Stroke 2013; 44: 2091 
[61] Lansberg MG, Straka M, Kemp S et al. MRI profile and response to endovascular reperfusion after stroke (DEFUSE 2): A prospective cohort study. Lancet Neurol 2012; 11: 860-867

[62] Lansberg MG, Cereda CW, Mlynash M et al. Response to endovascular reperfusion is not time-dependent in patients with salvageable tissue. Neurology 2015; 85: 708-714

[63] Wouters A, Lemmens R, Christensen S et al. Magnetic resonance imaging-based endovascular versus medical stroke treatment for symptom onset up to $12 \mathrm{~h}$. Int J Stroke 2016; 11: 127-133

[64] Gascou G, Lobotesis K, Machi P et al. Stent retrievers in acute ischemic stroke: Complications and failures during the perioperative period. AJNR 2014; 35: 734-740

[65] Mokin M, Fargen KM, Primiani CT et al. Vessel perforation during stent retriever thrombectomy for acute ischemic stroke: technical details and clinical outcomes. J Neurointerv Surg 2016, doi:10.1136/ neurintsurg-2016-012707
[66] Chen H, Liu N, Li Y et al. Mismatch of low perfusion and high permeability predicts hemorrhagic transformation region in acute ischemic stroke patients treated with intra-arterial thrombolysis. Sci Rep 2016; 6: 27950

[67] Coutinho JM, Liebeskind DS, Slater LA et al. Mechanical thrombectomy for isolated M2 occlusions: A post hoc analysis of the STAR, SWIFT, and SWIFT PRIME studies. AJNR 37: 2016; 667-672

[68] Singer OC, Berkefeld J, Nolte $\mathrm{CH}$ et al. Mechanical recanalization in basilar artery occlusion: The ENDOSTROKE study. Ann Neurol 2015; 77: 415-424

[69] van der Hoeven EJRJ, Schonewille WJ, Vos JA et al. The Basilar Artery International Cooperation Study (BASICS): study protocol for a randomised controlled trial. Trials 2013; 14: 200 